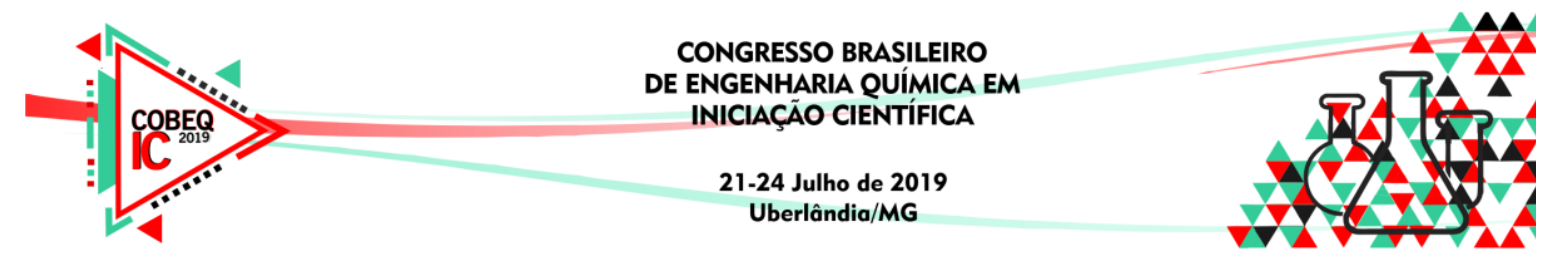

\title{
SINDRI: UMA NOVA FERRAMENTA COMPUTACIONAL PARA CÁLCULOS DE PROPRIEDADES TERMODINÂMICAS E DE EQUILÍBRIO LÍQUIDO-VAPOR A PARTIR DE EQUAÇÕES DE ESTADO CÚBICAS
}

\author{
M. B. F. SILVA ${ }^{1}$ e N. S. EVANGELISTA ${ }^{1}$ \\ ${ }^{1}$ Universidade Federal de Lavras, Departamento de Engenharia \\ E-mail para contato: marcusbfs@gmail.com
}

\begin{abstract}
RESUMO - É de grande interesse, para estudantes e profissionais de engenharia química, o conhecimento de propriedades termodinâmicas de substâncias puras e de misturas, além de dados de equilíbrio entre fases. Devido a alta quantidade e complexidade de sistemas de interesse industrial, a determinação de todas essas variáveis de interesse por vias experimentais se torna impraticável. Assim, o presente trabalho apresenta uma nova ferramenta computacional para a estimativa de propriedades termodinâmicas de substâncias puras/misturas e de dados de equilíbrio líquido-vapor. A ferramenta possui diversas equações de estado cúbicas, sendo elas utilizadas como modelos para todos os cálculos. O software faz o uso de uma interface gráfica simples e intuitiva, permitindo a alteração da equação de estado cúbica a qualquer momento, fazendo com que a comparação entre os dados seja rápida e efetiva. A ferramenta desenvolvida pode ser utilizada tanto para fins didáticos quanto práticos, sendo disponibilizada de maneira inteiramente gratuita $\mathrm{e}$ com o código aberto.
\end{abstract}

\section{INTRODUÇÃO}

Ao longo dos anos, a simulação de processos tem sido imprescindível para o projeto e otimização de processos nas indústrias química, petroquímica, farmacêutica, alimentícia entre outras. A base para a simulação de qualquer processo é um conjunto de propriedades termodinâmicas dos componentes puros e das misturas envolvidas nas diversas operações unitárias existentes (Poling et al., 2001). Para o projeto de equipamentos de separação envolvendo múltiplas fases (p. ex., destilação, absorção, esgotamento etc.), são necessárias também informações acerca do equilíbrio líquido-vapor dos componentes envolvidos (Prausnitz et al., 1999).

A velocidade com que o conhecimento das propriedades é demandado supera a velocidade com que elas são disponibilizadas na literatura. Isto é particularmente agravado para misturas, cujas propriedades variam drasticamente em virtude da natureza química e da proporção entre os seus constituintes. Assim, a lacuna entre a taxa com que novos compostos são sintetizados e a taxa com que os dados experimentais de suas propriedades termodinâmicas são coletados tende a aumentar indefinidamente com o tempo (Poling et al., 2001). Para contornar este problema, torna-se necessária a aplicação de modelos termodinâmicos, a exemplo das equações de estado cúbicas (EDEC). Tais equações se destacam por apresentarem 


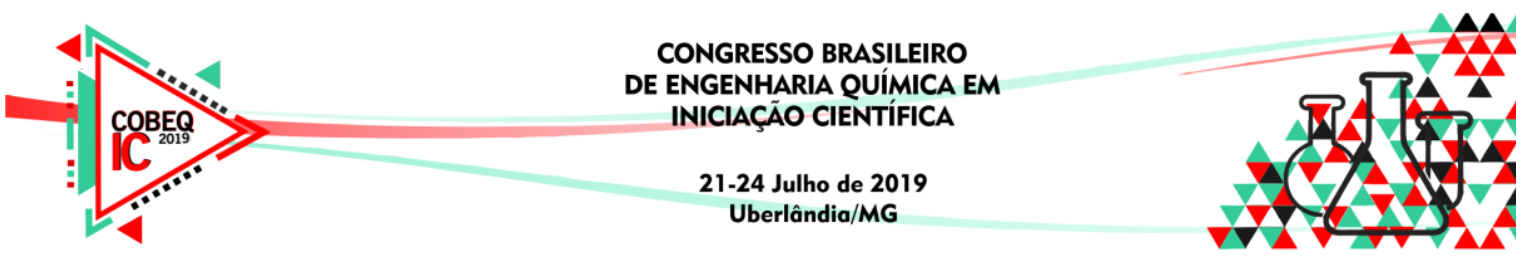

uma estrutura matemática simples e por gerarem resultados satisfatórios para uma grande variedade de substâncias de interesse industrial, o que as torna adequadas para muitas aplicações.

Em conjunto com o avanço dos modelos, houve o progresso tecnológico. Nas últimas décadas, programas e softwares computacionais foram desenvolvidos com o objetivo de facilitar a estimativa de propriedades termodinâmicas e de dados de equilíbrio líquido-vapor. Dentre esses softwares, destacam-se os programas de código-fechado PRZ e VLMU (Sandler, 1999), PRPURE e PRMIX (Elliot e Lira, 1999), Thermosolver (Koretsky, 2007) e VRTherm (VRTech, 2004); e os programas de código-aberto XSEOS (Castier e Amer, 2011) e OCTOPUS (Evangelista et al., 2016). Apesar da grande utilidade destes softwares, dificuldades são encontradas ao utilizá-los. A maioria dos softwares empregados nesta área são comerciais, o que dificulta o acesso de novos usuários. Em adição a isto, os softwares de código fechado impossibilitam o conhecimento dos algoritmos aplicados, aspecto que impede que estudantes, engenheiros e pesquisadores de tirarem proveito do código em busca de avanços em suas áreas. Além disso, alguns destes programas são limitados em relação à quantidade de EDEC disponíveis e por não emitirem relatórios e gráficos com os resultados.

Ante o exposto, o presente trabalho tem como finalidade apresentar um software de código aberto, com interface gráfica e estruturado com o paradigma de orientação a objetos, que proporciona ao usuário um acesso à um banco de dados editável, contendo centenas de substâncias de interesse industrial. O software possui diversas equações de estado cúbicas, que são utilizadas na estimativa de propriedades termodinâmicas e nos cálculos de equilíbriolíquido vapor para substâncias puras ou sistemas multicomponentes. O software desenvolvido é disponibilizado de forma totalmente gratuita, de modo a contribuir para a comunidade de cientistas e engenheiros, podendo ser utilizado para fins educacionais ou de pesquisa.

\section{EQUAÇÕES DE ESTADO CÚBICAS IMPLEMENTADAS}

Utilizando a linguagem Python na versão 3.7, foram implementadas 19 equações de estado cúbicas para o cálculo de propriedades termodinâmicas de substâncias puras e misturas, a saber: van der Waals (1890), Redlich e Kwong (1949), Wilson (1964), Soave (1972), Peng e Robinson (1976), Patel e Teja (1982), Péneloux et al. (1982), Adachi et al. (1983), Mathias e Copeman (1983), Soave (1984), Adachi et al. (1985), Stryjek e Vera (1986), Twu et al. (1995), Tsai e Chen (1998), Ahlers e Gmehling (2001), Gasem et al. (2001) (três equações) e Coquelet et al. (2004). Com exceção das equações de Tsai e Chen (1998) e de Coquelet et al. (2004), todas as outras foram implementadas conforme constam nos livros de Poling et al. (2001) e de Gmehling et al. (2012).

\section{ESTRUTURA DO SOFTWARE}

\subsection{Banco de Dados}

Para a estimativa de propriedades termodinâmicas, é necessário um banco de dados contentando propriedades básicas das substâncias como temperatura e pressão no ponto crítico, fator acêntrico e parâmetros para cálculo da capacidade calorífica. $\mathrm{O}$ banco de dados presente no software foi gerado a partir dos dados reportados por Poling et al. (2001) e contém dados de 469 substâncias, incluindo compostos orgânicos e inorgânicos. O banco de dados apresenta 


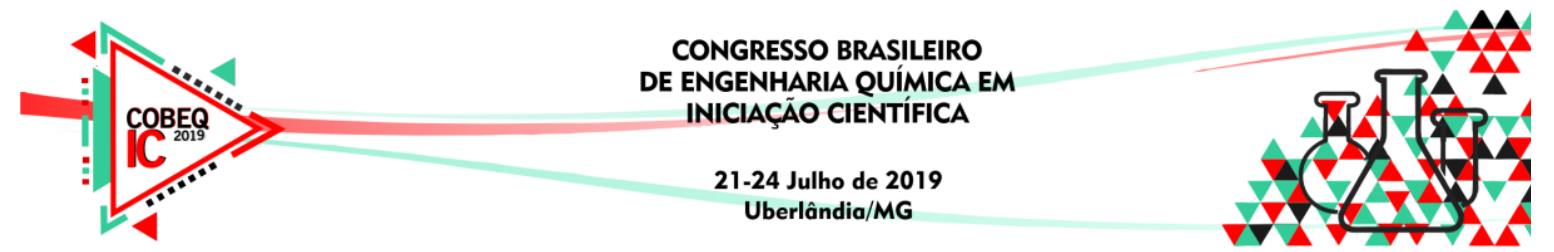

uma interface de interação do usuário, que o permite pesquisar as substâncias por meio de seu nome, fórmula, ou número CAS, podendo também editar qualquer propriedade das substâncias já existentes ou adicionar uma nova substância ao banco de dados.

\subsection{Cálculo de Propriedades Termodinâmicas}

O software desenvolvido é capaz de calcular, utilizando qualquer uma das equações de estado cúbicas disponíveis, propriedades termodinâmicas para substâncias puras e misturas. Na Figura 1, é exemplificado o uso da interface de cálculo. Ressalta-se que, para substâncias puras, o software calcula a pressão de vapor pela EDEC selecionada e pelas equações de Antoine (1888), Lee e Kesler (1975) e Ambrose e Walton (1989), possibilitando uma rápida comparação de resultados entre os modelos. O software também é capaz de gerar relatórios e diagramas relacionando propriedades termodinâmicas de substâncias puras, o que auxilia no entendimento do sistema por meio da visualização de seu comportamento por toda a faixa de saturação. Dentre os gráficos, citam-se os diagramas de pressão por volume, entalpia por entropia e pressão de saturação por temperatura.z

Figura 1 - Propriedades termodinâmicas do hexano, a $350 \mathrm{~K}$ e 1 bar, calculadas pela EDEC de Peng-Robinson (1976).

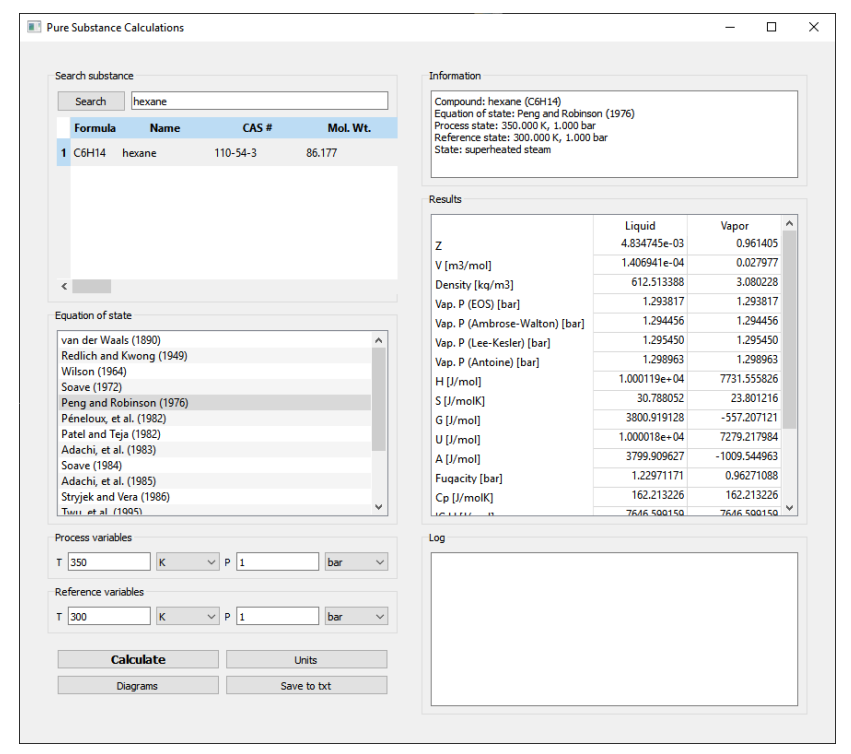

\subsection{Equilíbrio Líquido-Vapor para Sistemas Multicomponentes}

O equilíbrio líquido-vapor multicomponente é modelado por meio do método simétrico $\Phi-\Phi$. Foram utilizados os algoritmos propostos por Michelsen e Mollerup (2008) para a resolução dos problemas de ELV mais comuns: ponto de bolha, ponto de orvalho, e flash PT. Para a utilização das EDEC no cálculo de misturas, foi implementada a regra de mistura clássica por ser amplamente usada e conter um parâmetro que pode ser ajustado para melhor adequar os resultados estimados aos experimentais.

A Figura 2 mostra a interface para os cálculos de equilíbrio líquido-vapor de sistemas multicomponentes. O usuário, caso deseje, pode selecionar uma nova EDEC para a realização dos cálculos, o que facilita a análise comparativa entre os modelos. Além disso, o usuário tem 


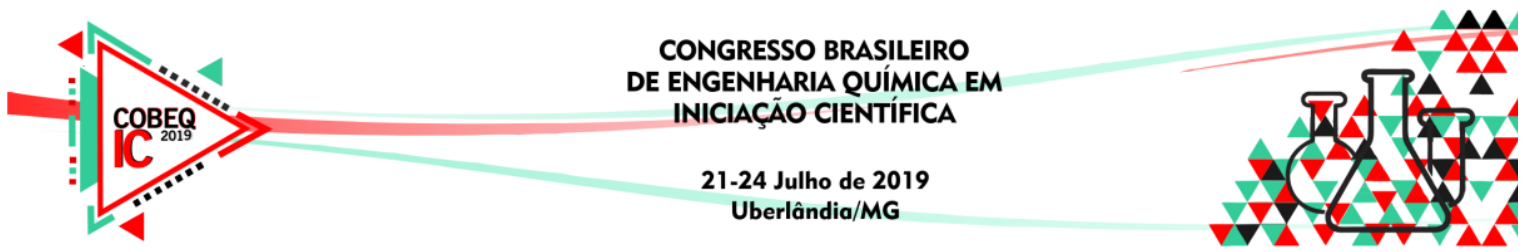

a opção de importar dados experimentais para os sistemas de interesse, havendo a possibilidade de plotar tais dados para a validação dos modelos.

Figura 2 - Cálculo flash e geração de dados de equilíbrio para o sistema pentano-hexano, pela EDEC de Soave (1972).
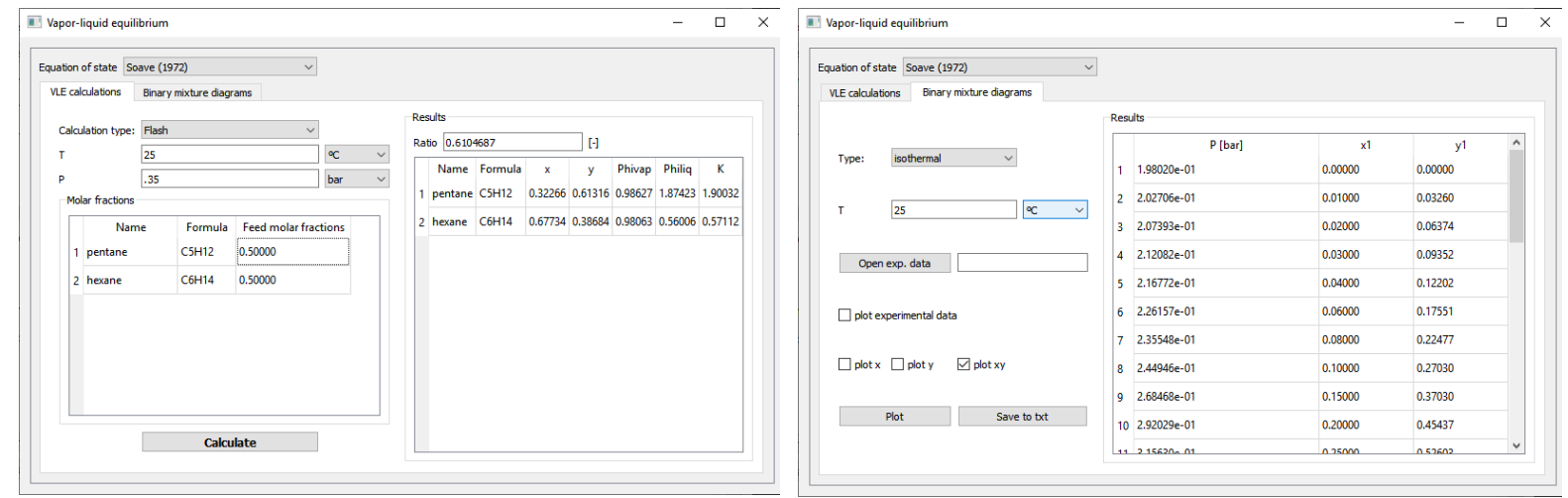

\section{APLICAÇÕES}

\subsection{Cálculo Flash}

O cálculo flash é um problema recorrente em processos de separação. A Tabela 1 mostra a resolução do cálculo flash para um sistema ternário contendo acetona (1), acetonitrila (2) e nitrometano (3) a $80^{\circ} \mathrm{C}$ e $110 \mathrm{kPa}$, com a composição global $z_{1}=0,45 ; z_{2}=0,35$ e $z_{3}=0,20$. Os resultados foram calculados com o programa apresentado e o software VRTherm. Em ambos os programas, a equação de estado cúbica utilizada foi a de Peng e Robinson (1976). A partir dos resultados apresentados, percebe-se a confiabilidade dos cálculos de equilíbrio efetuados pelo software. Em comparação com o VRTherm, o software desenvolvido se destaca por apresentar os coeficientes de fugacidade de cada substância para ambas fases, tal como a fração da alimentação do flash que é evaporada.

Tabela 1 - Resultados do cálculo flash para o sistema estudado

\begin{tabular}{|c|c|c|c|c|c|c|}
\hline \multirow{2}{*}{ Componente } & \multicolumn{2}{|c|}{ Programa desenvolvido } & \multicolumn{2}{|c|}{ VRTherm } & \multirow{2}{*}{$\begin{array}{c}\text { erro } \\
\text { relativo (\%) }\end{array}$} & $\begin{array}{c}y-\text { erro } \\
\text { relativo (\%) }\end{array}$ \\
\cline { 2 - 7 } & $x$ & $y$ & $x$ & $y$ & 0,01 & 0,40 \\
\hline Acetona & 0,23492 & 0,46822 & 0,23495 & 0,47012 & $-0,17$ \\
\hline Acetonitrila & 0,38736 & 0,34684 & 0,39016 & 0,34624 & 0,72 & $-0,70$ \\
\hline Nitrometano & 0,37772 & 0,18494 & 0,37489 & 0,18364 & $-0,75$ & \\
\hline
\end{tabular}

\subsection{Diagrama para Equilíbrio de Fases}

O software desenvolvido é capaz de gerar gráficos de equilíbrio (isotérmicos ou isobáricos) para sistemas binários. Para ilustrar este procedimento, foi analisado o sistema hexano (1) e ciclohexano (2), modelados pelas equações de estado cúbica de Peng e Robinson (1976) e de Stryjek e Vera (1986).

A fim de facilitar a visualização e comparação entre modelo e dados experimentais, o software permite a leitura de um arquivo texto contendo os dados de equilíbrio do sistema 


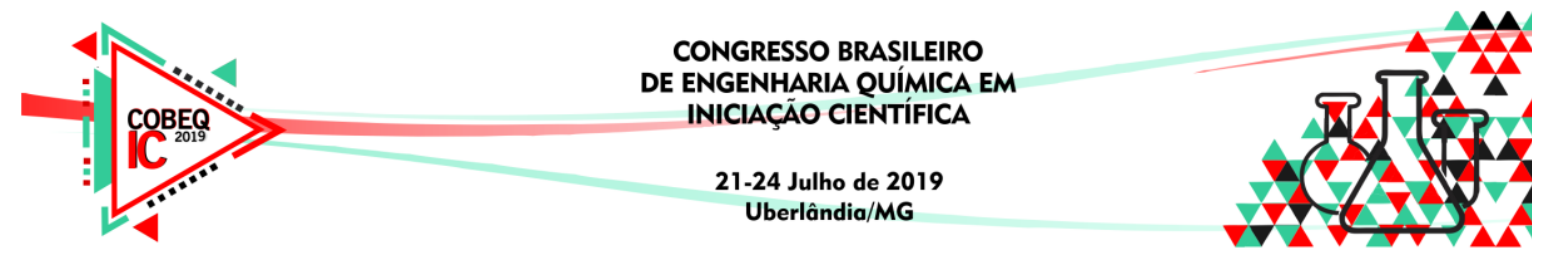

analisado. Os dados de equilíbrio líquido-vapor obtidos por Susarev e Chen (1963) para a mistura hexano-ciclohexano a $343,15 \mathrm{~K}$ foram utilizados para comparar as estimativas das duas equações aplicadas. A Figura 3 apresenta os gráficos gerados pelo software, juntamente com os dados experimentais supracitados.

Figura 3 - Dados do equilíbrio líquido-vapor para o sistema hexano-ciclohexano estimados pelo software
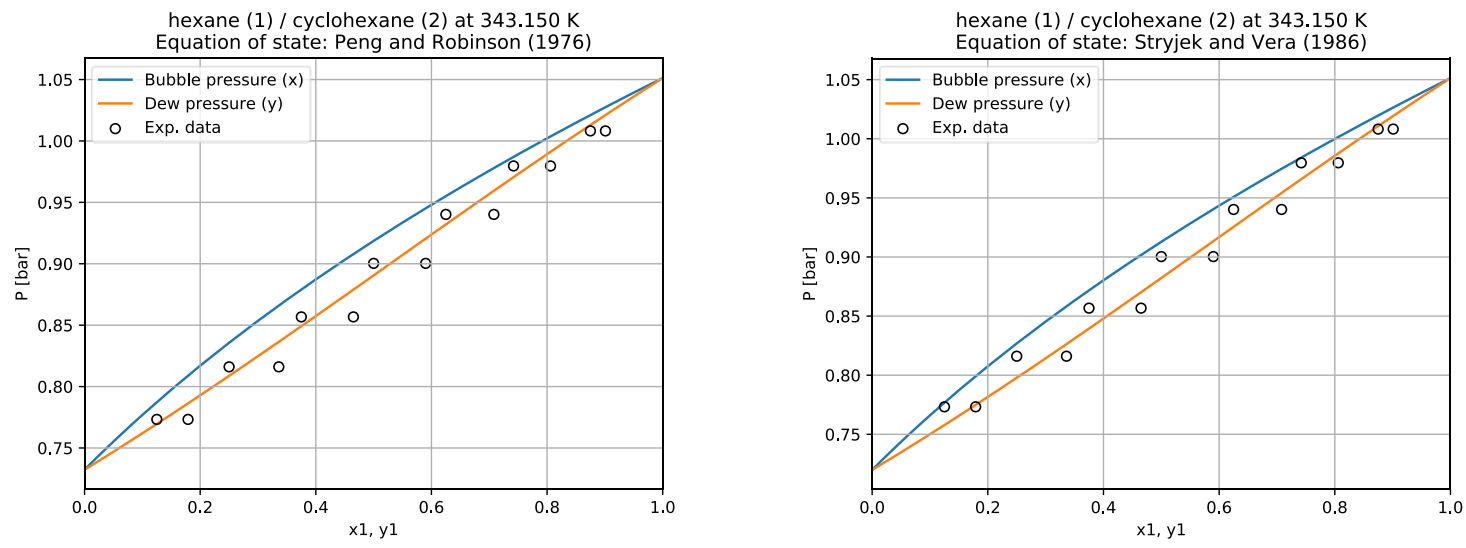

É observado que a EDEC de Stryjek e Vera (1986) se adequa melhor aos dados experimentais, principalmente na curva de orvalho; este fato é esperado, dito que esta equação é um aprimoramento da EDEC de Peng e Robinson (1976). Ressalta-se que as equações implementadas no software possuem um parâmetro de interação binária na regra de mistura, que pode ser definido pelo usuário por meio da interface gráfica. Este parâmetro pode ser ajustado para a obtenção de dados mais fidedignos aos experimentais.

\section{CONCLUSÕES}

O presente trabalho apresentou uma nova ferramenta computacional para a estimativa de propriedades termodinâmicas e dados equilíbrio líquido-vapor pelo método simétrico $\Phi-\Phi$. Há uma variedade de equações de estado cúbicas, permitindo ao usuário uma fácil comparação entre os resultados e, consequentemente, auxiliando-o na escolha da equação propícia ao seu objetivo. Por ser disponibilizada de forma totalmente gratuita e de código aberto, acredita-se que esta ferramenta pode ser útil em aplicações de ensino e de pesquisa para estudantes, engenheiros e profissionais. O código fonte e o instalador do software, juntamente com o manual de instalação, podem ser encontrados no repositório virtual www.github.com/mrcsbrn/Sindri.

\section{REFERÊNCIAS}

AMBROSE, D.; WALTON, J. Vapour pressures up to their critical temperatures of normal alkanes and 1-alkanols. Pure Appl. Chem., v. 61, n. 8, p. 1395-1403, 1989.

ANTOINE, C. Thermodynamique, Tensions des Vapeurs: Nouvelle Relation entre les Tensions et les Temperatures. C. r. hebd. séances Acad. sci., v. 107, p. 681-684-837, 1888. 


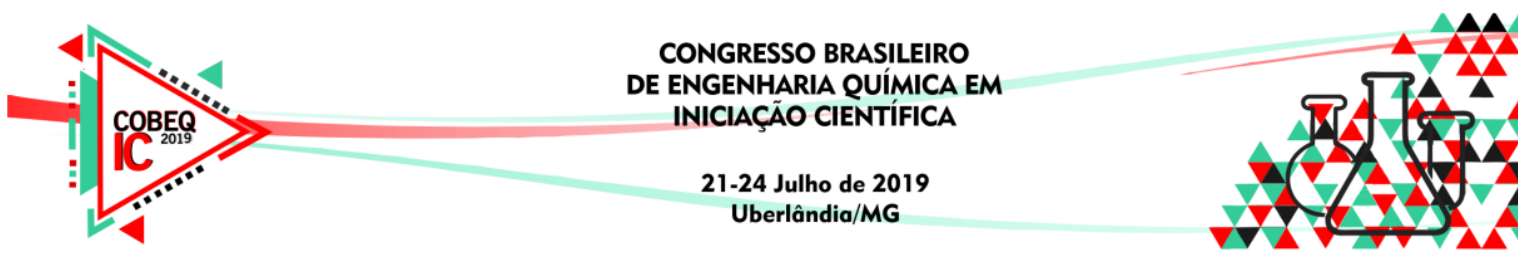

CASTIER, M.; AMER, M. M. XSEOS: An evolving tool for teaching chemical engineering thermodynamics. Education for Chemical Engineers, v. 6, n. 2, p. e62-e70, 2011.

COQUELET, C.; CHAPOY, A.; RICHON, D. Development of a new alpha function for the Peng-Robinson equation of state: Comparative study of alpha function models for pure gases (natural gas components) and water-gas systems. Int. J. Thermophys., 2004.

ELLIOTT, J. R.; LIRA, C. T. Introductory Chemical Engineering Thermodynamics. 1. ed. New Jersey: Prentice-Hall, 1999

EVANGELISTA, N. S.; DO CARMO, F. R.; DE SANT'ANA, H. B. OCTOPUS: uma nova ferramenta computacional para estimativa de propriedades físicas e químicas de compostos orgânicos moleculares. XXI Congresso Brasileiro de Engenharia Química. Fortaleza, Ceará. 2016.

GMEHLING, J; KLEIBER, M; KOLBE, B.; RAREY, J. Chemical Thermodynamics for Process Simulation. 1. ed. Weinheim: Wiley-VCH, 2012

KORETSKY, M. D. Termodinâmica para Engenharia Química. 1. ed. Rio de Janeiro: LTC, 2007.

LEE, B. I.; KESLER, M. G. A generalized thermodynamic correlation based on threeparameter corresponding states. AIChE Journal, v. 21, n. 3, p. 510-527, 1975.

MICHELSEN, M. L.; MOLLERUP, J. M. Thermodynamic Models: fundamentals \& computacional aspects. 2. ed. Holte: Tie-Line Publications, 2008.

PENG, D. Y.; ROBINSON, D. B. A new two-constant equation of state. Industrial \& Engineering Chemistry Fundamentals, v. 15, n. 1, p. 59-64, 1976.

POLING, B. E.; PRAUSNITZ, J. M.; O'CONNELL, J. P. The Properties of Gases and Liquids. New York: Mcgraw-Hill, 2001.

PRAUSNITZ, J. M.; LICHTENTHALER, R. N.; AZEVEDO, E. G. Molecular Thermodynamics of Fluid-Phase Equilibria. 3. ed. New Jersey: Prentice-Hall, 1999.

SANDLER, S. I. Chemical and Engineering Thermodynamics. 3. ed. New York: John Wiley \& Sons, 1999.

STRYJEK, R.; VERA, J. H. PRSV: An improved Peng-Robinson equation of state for pure compounds and mixtures. The Can. J. of Chem. Eng., v. 64, n. 2, p. 323-333, 1986.

SUSAREV, M. P.; CHEN, S. T. The Benzene-n-Hexane-Cyclo-Hexane System. Russ. J. Phys. Chem. 37. 1963.

TSAI, Jung-Chin; CHEN, Yan-Ping. Application of a volume-translated Peng-Robinson equation of state on vapor-liquid equilibrium calculations. Fluid phase equilibria, v. 145, n. 2, p. 193-215, 1998.

VRTherm Thermodynamic Package. VRTech Tecnologias Industriais LTDA. 2004. Disponível em <http://www.iise.ltd/pt/vrtherm> 